\title{
A novel performance evaluation paradigm for automated video surveillance systems
}

\author{
Chung-Hao Chen ${ }^{1 *}$, Yi Yao ${ }^{2}$, Andreas Koschan ${ }^{3}$, Mongi Abidi ${ }^{3}$
}

1 Department of Electrical and Computer Engineering, Old Dominion University, Norfolk, USA

2 General Electric, USA

3 Imaging, Robotics, and Intelligent Systems Laboratory, The University of Tennessee, Knoxville, USA

Received 24 June 2011 ; accepted 04 December 2011

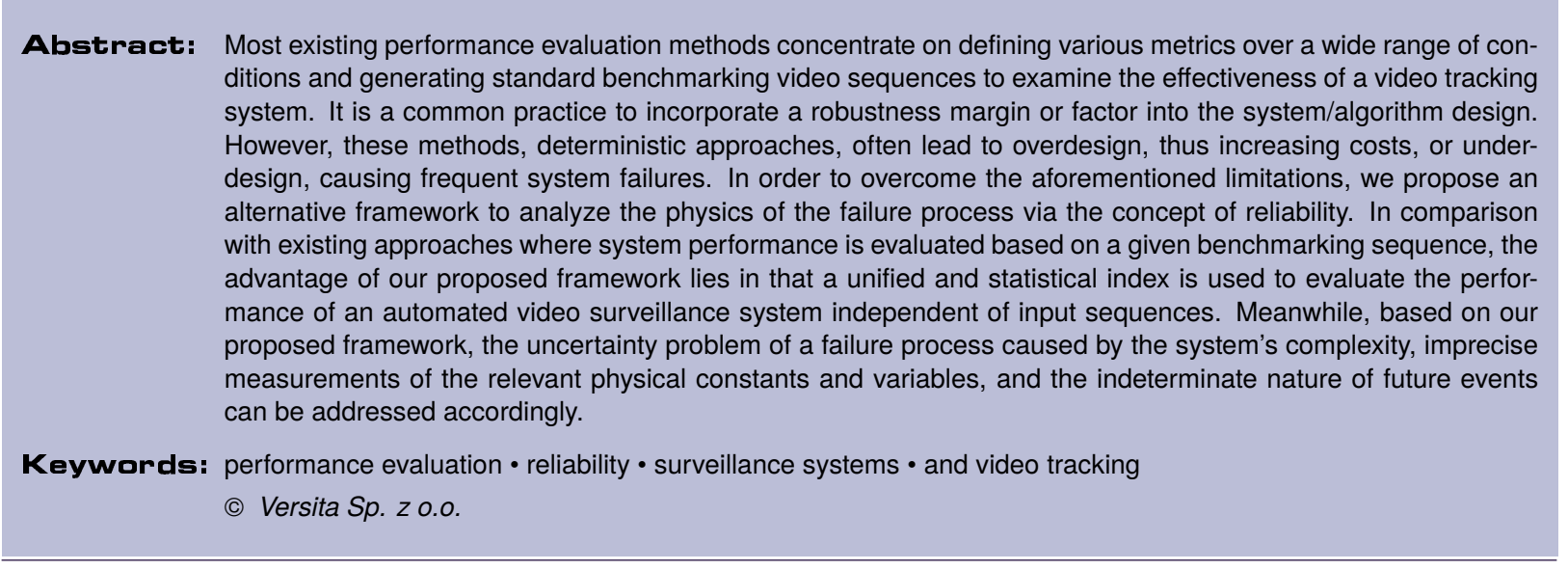

\section{Introduction}

Automated video surveillance systems have been widely studied in the past decade. Because of its promise that it can monitor an area without human intervention all times, its applications can cover crime prevention [16], pre-emptive interest

\footnotetext{
*E-mail: cxchen@odu.edu
} 
protection [2], national security [23], etc. However, even though results of most present automated video surveillance systems are efficient in some cases, the evaluation of the robustness of an automated video surveillance system remains difficult given the requirement that a system should operate in all times and under varying conditions as, for example, changing weather conditions, number of subjects in the scene, and illuminations. Thus, the issue of evaluating the performance of automated video surveillance systems has become increasingly important.

One conventional approach $[6,11]$ to performance evaluation is to generate ground truth from pre-recorded video sequences. List et al. [15] pointed out that the manual generation of ground truth is a time-consuming task and, thus, inevitably error prone. Performance evaluation algorithms based on comparisons with ground truth can be further classified according to the type of metrics employed [10,17-20]. In the case of foreground-background segmentation, metrics are useful to assess the overall segmentation quality on frame-to-frame basis but they do not provide an evaluation of individual object segmentation, because they are restricted to pixel-level discrepancy between the detected foreground and the ground truth [14]. Other smaller-scale evaluation frameworks include comprehensive approach [13], frame-based and object-based metrics [1], Label and Size Based Evaluation Measure (LSBEM) [21], or Online parameter based approach $[4,24]$. The aforementioned methods, deterministic approaches, usually reserve a robustness margin or factor, such as two or three times the expected number of subjects to be tracked or the strength of illumination, in the system design. This often results in overdesign, thus increasing costs, or underdesign causing frequent system failures from unexpected disturbances. Particularly, none of existing frameworks is able to provide a quantitative alternative to answer the question of how to improve the performance with the existing system, e.g. a redundant system setup, after they evaluate the performance of the desired automated video surveillance system.

In this paper, we propose an alternative framework to analyze the failure process, examine the system reliability, and derive the average time duration between system failures for automated video surveillance systems. The proposed framework has the advantage of addressing the uncertainty of a failure process, which may be caused by the system complexity, imprecise measurements of the relevant physical constants and variables, and the indeterminate nature of future events. In addition, the proposed framework can provide a guideline of how to improve the reliability of an automated video surveillance system.

The remainder of this paper is organized as follows. Section 2 introduces the concept of reliability. Section 3 describes our proposed framework for evaluating the reliability of automated video surveillance systems and determining how to improve its overall reliability. Section 4 illustrates our experimental results and Section 5 concludes the paper.

\section{Reliability}

Reliability [7, 22] is defined as the probability that a component or system can perform a required function for a given period of time, $t$, when used under stated operating conditions [9]. In other words, it is the probability of a non-failure over time. In terms of the interpretation of quality, reliability is concerned with how long the system continues to function once it becomes operational. A poor-quality system will likely have poor reliability, and a high-quality system will have a high reliability. To define the reliability in a system, three definitions must be made specific: (1) failures should be defined relative to the function being performed by the system; (2) the unit of time must be identified; (3) the system should be observed under normal performance such as environment, design loads, and operating conditions. The reliability can be expressed as

$$
R(t)=\operatorname{Pr}\{T \geq t\}
$$

where $\mathrm{T}$ represents the time to failure of the system and $T \geq 0 . R(t) \geq 0, R(0)=1$, and $\lim _{t \rightarrow \infty} R(t)=0$. If we define

$$
F(t)=1-R(t)=\operatorname{Pr}\{T<t\}
$$

where $F(0)=0$ and $\lim _{t \rightarrow \infty} F(t)=1$, then $F(t)$ is the probability that a failure occurs before time $t$. We will refer to $R(t)$ as the reliability function and $F(t)$ as the cumulative distribution function (CDF) of failure distribution, and

$$
f(t)=\frac{d F(t)}{d t}=-\frac{d R(t)}{d t}
$$


as the probability density function (PDF). Thus, the failure rate function, $\lambda(t)$, provides an instantaneous rate of failure at time $t$, which is defined as

$$
\lambda(t)=-\frac{d R(t)}{d t} \cdot \frac{1}{R(t)}
$$

A suggestion of a failure rate function is shown in Figure 1. Systems having the failure rate function in bathtub curve experience decreasing failure rates early in their life circle (burn-in), followed by a nearly constant failure rate (useful life), followed by an increasing failure rate (wear-out). In general, burn-in period in the system is caused by defective parts, contamination, or poor quality control. It can be reduced by quality control, screening, burn-in testing, and acceptance testing. Useful life period in the system is cased by random loads or human error. It can be reduced by component redundancy and excess strength. Aging and fatigue cause wear-out period in the system. It can be reduced by components replacement, preventive maintenance, and improved technology. Based on Equation (4), we can derive

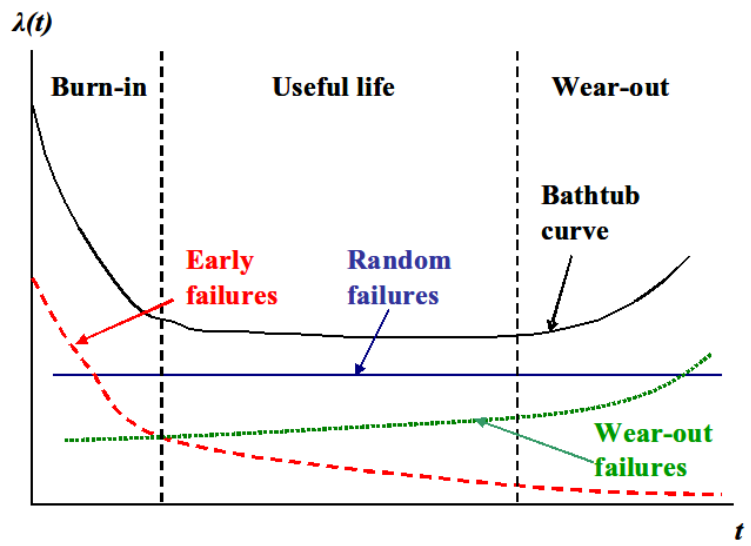

Figure 1. Illustration of failure rate function.

the reliability model,

$$
R(t)=\exp \left[-\int_{0}^{t} \lambda\left(t^{\prime}\right) d t^{\prime}\right]
$$

and the mean time to failure (MTTF),

$$
M T T F=E(T)=\int_{0}^{\infty} t f(t) d t .
$$

\section{Proposed framework}

Often governmental and private contract specifications require that each function being performed by the system must have a 90 percent or better reliability over a designed time. Thus, the objective of our proposed framework is to derive the reliability function, $R(t)$, of the automated video surveillance system based on collected data and defined metrics. Furthermore, we can establish a unified probability describing the chance of the system running functionally over a designed time in a given environment.

In doing so, we perform a statistical test in order to accept or reject the hypothesis that the observed failure times come from a specified distribution. In general, fitting a theoretical distribution is preferred over empirically developing a model, namely nonparametric model, because empirical models do not provide sufficient information beyond the range of the sample data [9]. In reliability engineering the tails of the distribution are of most interest. Often the failure process is a result of some physical phenomena that can be associated with a particular distribution. If the sample is consistent with a theoretical distribution, then much stronger results are possible based on the properties of the theoretical distribution. Figure 2 illustrates our proposed reliability assessment for automated video surveillance systems. 
In Figure 2, test conditions represent an environment where the system is expected to operate. Conditions can be set to generic terms such as a predefined indoor illumination, randomly or regularly moving multi-object, etc. Once test conditions are established, we can perform iterative experiments to sample failure times, which of each failure time indicate how long the system can function in a predefined measurement. For example, the measurement is set to two randomly walking subjects should be tracked simultaneously by the automated video surveillance. If not, record the time period from the beginning of this experiment to the failure. Afterwards, we use Chi-Square goodness of fit test to

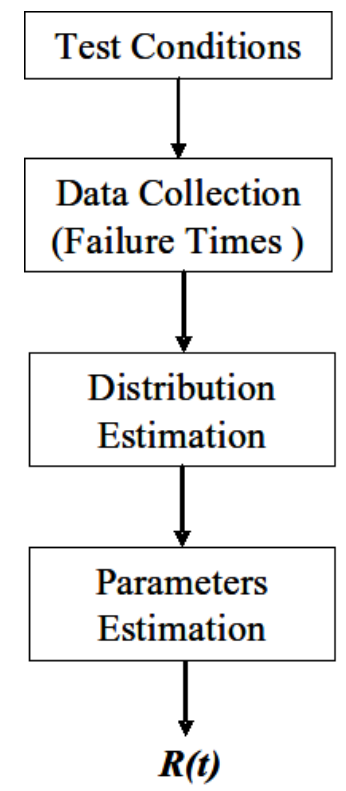

Figure 2. Illustration of our proposed framework for assessing the reliability of automated video surveillance systems.

determine which theoretical distribution can most appropriately represent the collected failure times because it does not have the restriction that location, scale, and shape parameters cannot be estimated from the data, as compared with Kolmogorov-Smirnov goodness of fit test. Chi-Square goodness of fit test compares a null hypothesis $\left(H_{0}\right)$ with an alternative hypothesis $\left(H_{1}\right)$ having the following form:

$H_{0}$ : the failure times came from the specified distribution.

$H_{1}$ : the failure times did not come from the specified distribution.

In this paper, four theoretical distributions, exponential, weibull, normal, and lognormal are performed to describe the failure process of the system. A failure distribution that has a constant failure rate (CFR) is called an exponential failure law $[7,22]$. Failures due to completely random or chance events will follow this distribution. It should dominate during the useful life of a system or component. The exponential distribution is one of the most common reliability distributions in this category. Weibull, normal, and lognormal distributions have failure rate functions that are not constant over time, thus providing a necessary alternative to the exponential failure law.

In general, $H_{0}$ is accepted if the value of its test statistic is smaller than its critical value. In the meantime, parameters of each distribution are estimated from maximum likelihood estimators (MLEs). In particular, since Chi-Square goodness of fit test can only present if this data come from the specified distribution, we have the likeliness that multiple theoretical distributions are accepted [22]. Thus, least-square curve fitting is used to determine which distribution is the best fit in the pool of accepted theoretical distributions. In general, the bigger the value of the least-square curve fitting (index of fit), the better fit it is [22]. Once parameters of the specified distribution are derived, we can obtain the reliability model of an automated video surveillance system, which is based on Equation (2), under tested conditions. 


\section{System reliability improvement}

Redundancy may play an important role in the design process, when individual component reliability has been established. Moreover, when it is inefficient to achieve the desired component reliability through inherent component design, redundancy may provide the only alternative. In addition, redundancy may allow for increased reliability against external environmental stress. Two or more components are in redundant configuration if all components must fail for the whole system to fail. If one or more components operate, the system continues to operate. Redundant component are represented by Figure 3. Thus, the reliability for this new system, $R_{s}(t)$, can be represented as

$$
\begin{aligned}
& \left.R_{s}(t)=P\left(E_{1}\right) \cup E_{2} \cup \cdots \cup E_{n}\right) \\
& =1-P\left(E_{1} \cup E_{2} \cup \cdots \cup E_{n}\right)^{C} \\
& =1-\left(1-R_{1}(t)\right)\left(1-R_{2}(t)\right) \ldots\left(1-R_{n}(t)\right), \\
& =1-\prod_{i=1}^{n}\left[1-R_{i}(t)\right],
\end{aligned}
$$

where $R_{i}(t)$ represents the reliability function of component $\mathrm{i}$ and $\mathrm{n}$ is the number of redundant components. $E_{n}$ is the event that component $\mathrm{n}$ does not fail and $P\left(E_{n}\right)$ is equal to $R_{n}(t)$. It is always true that

$$
R_{s}(t) \geq \max \left\{R_{1}(t), R_{2}(t), \ldots, R_{n}(t)\right\}
$$

since it must be less than the failure probability of the most reliable component.

\section{Experimental results}

In our indoor real-time surveillance environment with dimensions of $30 \mathrm{~m} \times 15 \mathrm{~m} \times 3 \mathrm{~m}$, one omnidirectional camera (IQeve3) is placed in the middle of the room to be monitored at a height of $3 \mathrm{~m}$. The camera is used to perform multiobject tracking. Cui's background differencing and radial profile analysis [5] are applied in this scenario for multi-object tracking. Omnidirectional images with a resolution of $320 \times 320$ pixels are obtained via an intranet connection at a maximum speed of 10 frames per second. The strength of illumination lied between 400 lux and 650 lux in our indoor environment.

\subsection{Goodness of fit and curving fitting}

During the experiments two subjects are randomly walking in the environment. Whenever the two subjects are not being tracked simultaneously by the system, we stop the experiment and record the failure time. This procedure is carried out repeatedly during the experiment. Figure 4 illustrates one video sequence from the experiments. In Figure 4, two tracked subjects, who are marked with two red circles, are walking back and forth in the monitored environment. At time elapse 115 seconds, the system can only track one person, which is indicated by only one red circle. Thus, the failure time of this experiment is 115 seconds. We repeat the experiment again and collect 40 failure times. Table 1 summarizes Chi-Square goodness of fit and the least-square curve fitting for four theoretical distributions when the level of significance is set to 0.05 .

In Table 1, only a Weibull distribution can represent the collected data. Since the estimated parameters for the Weibull distribution are $\beta=1.77$ and $\theta=122$ in this case, the estimated reliability model of Cui's object tracking algorithm under our test environment with two randomly walking subjects is

$$
R(t)=e^{-\left(\frac{t}{122}\right)^{1.77}}
$$

and the failure rate function is

$$
\lambda(t)=0.0145\left(\frac{t}{122}\right)^{0.77}
$$




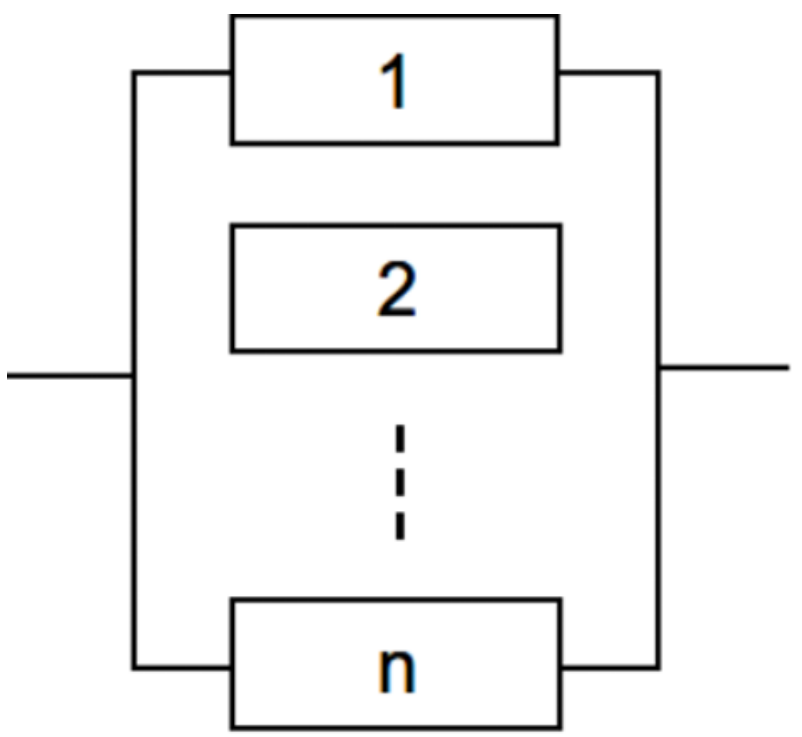

Figure 3. Reliability block diagram for components in redundancy.

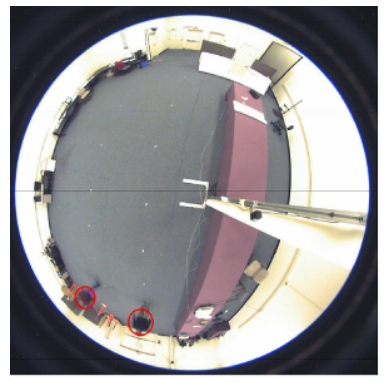

$t=0$ (seconds)

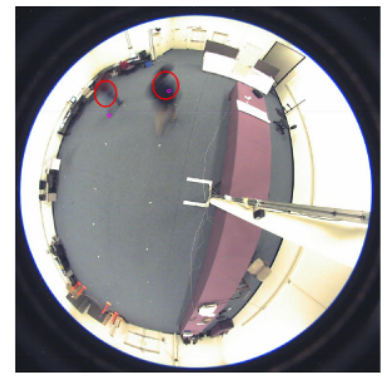

$t=15$ (seconds)

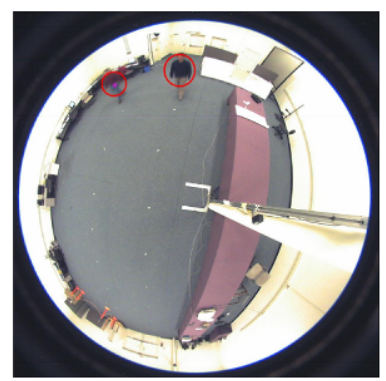

$t=65$ (seconds)

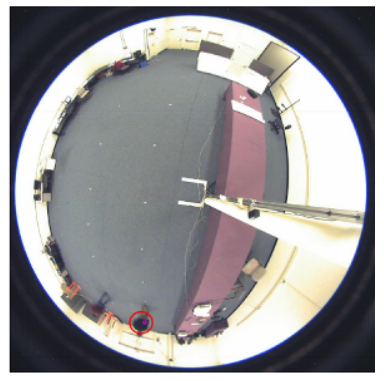

$t=115$ (seconds)

Figure 4. Illustration of one experimental video sequence.

In Weibull distribution, $\beta$ is referred to as the shape parameter. Its effect on the failure rate function can be seen in Figure 5 for several different values. Figure 5 shows that the failure rate function can be increasing or decreasing depending on the value of $\beta$. The value of the shape parameter $\beta$ provides insight into the behavior of the failure process. Table 2 summarizes this behavior. An increasing failure rate can increase at a decreasing rate (concave), increase at a constant rate (linear), or increase at an increasing rate (convex), depending on $\beta$ as shown in Table 2 .

Thus, since in this case $\beta$ is 1.7 , the increasing failure rate increases at a decreasing rate (concave). $\theta$ is a scale parameter that influences both the mean and the spread, or dispersion, of the distribution. The effect of $\theta$ on the failure

Table 1. Summary of goodness of fit for four theoretical distributions.

\begin{tabular}{|c|c|c|c|c|}
\hline Distribution & Test Statistic & Critical Value & $H_{0}$ & Index of Fit \\
\hline Exponential & 15.3 & 7.81 & Reject & 0.6 \\
\hline Weibull & 4.8 & 5.9 & Accept & 0.92 \\
\hline Normal & 7.24 & 5.9 & Reject & 0.68 \\
\hline Lognomal & 10 & 5.9 & Reject & 0.65 \\
\hline
\end{tabular}


Table 2. Weibull shape parameter.

\begin{tabular}{cc}
\hline Value & Property \\
\hline \hline $0<\beta<1$ & Decreasing failure rate \\
$\beta=1$ & Exponential distribution \\
$1<\beta<2$ & Concave \\
$\beta=2$ & Rayleigh distribution \\
$\beta>2$ & Convex \\
$3 \leq \beta \leq 4$ & Approaches normal distribution \\
\hline
\end{tabular}

rate function is illustrated in Figure 6 for several different values. In Figure 6, the slope of the hazard rate decreases as $\theta$ increases. In other words, as $\theta$ increases, the reliability increases at a given point in time. This parameter $\theta$ is also called the characteristic life, and it has units identical to those of the failure time.

Based on Equation (9), we can estimate the design life for a desired $90 \%$ reliability in our test environment with two randomly walking subjects by $t=122(-\ln 0.9)^{\frac{1}{1.77} \cong 34}$ (seconds).

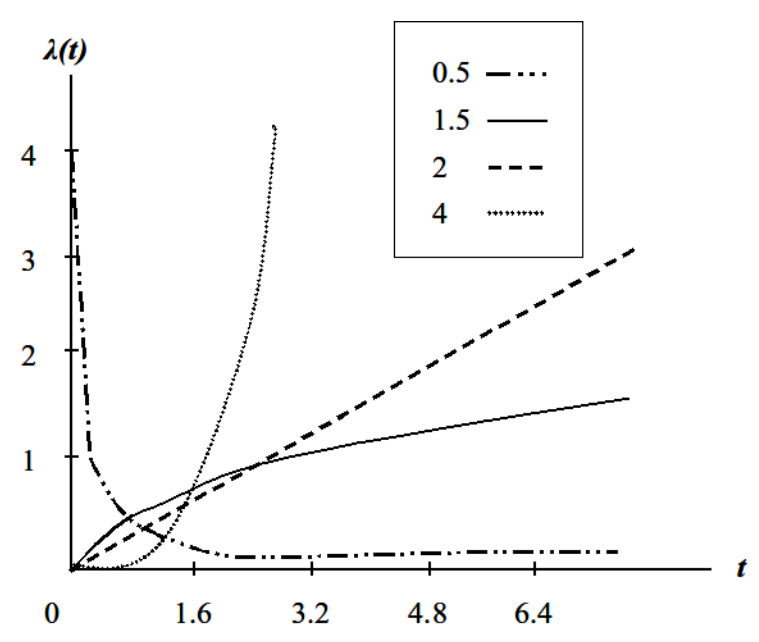

Figure 5. Illustration of the effect of $\beta$ on the Weibull failure rate curve over time.

\section{Redundancy with Weibull}

As mentioned before, redundancy may play an important role in the design process, when individual component reliability has been established. Moreover, when it is inefficient to achieve the desired component reliability through inherent component design, redundancy may provide the only alternative. In our case, the reliability function had been derived, as shown in Equation (9). If two identical (and independent) components are used to form a redundant system (both must fail for the system to fail), according to Equation (7), the system reliability $R_{s}(t)$ is represented as

$$
1-[1-R(t)]^{2}
$$

and $R(t)=e^{-\left(\frac{t}{122}\right)^{1.77}}$. Thus,

$$
R_{s}(t)=2 e^{-\left(\frac{t}{122}\right)^{1.77}}-e^{-2\left(\frac{t}{122}\right)^{1.77}}
$$




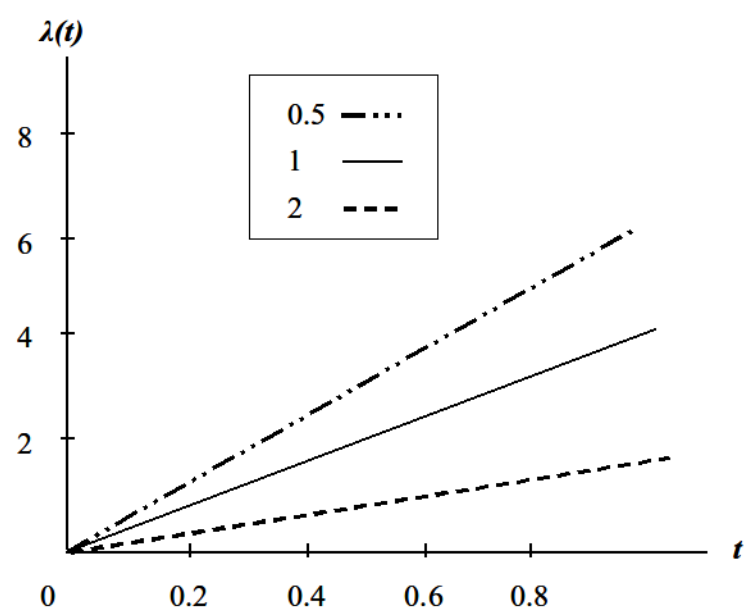

Figure 6. Illustration of the effect of $\theta$ on the Weibull failure rate curve over time.

In particular, the failure rate function of the system formed from two redundant Weibull components can be represented as

$$
\begin{array}{r}
\lambda_{s}(t)=-\frac{d R_{s}(t)}{d t} \cdot \frac{1}{R_{s}(t)} \\
=\frac{\beta}{\theta}\left(\frac{t}{\theta}\right)^{\beta-1} \frac{2-2 e^{-\left(\frac{t}{0}\right)^{\beta}}}{2-e^{-\left(\frac{t}{\theta}\right)^{\beta}}},
\end{array}
$$

where

$$
\frac{d R_{s}(t)}{d t}=2 e^{-\left(\frac{t}{\theta}\right)^{\beta}}\left[-\frac{\beta}{\theta}\left(\frac{t}{\theta}\right)^{\beta-1}\right]-e^{-2\left(\frac{t}{\theta}\right)^{\beta}}\left[-\frac{2 \beta}{\theta}\left(\frac{t}{\theta}\right)^{\beta-1}\right]=-\frac{\beta}{\theta}\left(\frac{t}{\theta}\right)^{\beta-1} e^{-\left(\frac{t}{\theta}\right)^{\beta}}\left[2-2 e^{-\left(\frac{t}{\theta}\right)^{\beta}}\right]
$$

Due to Equation (14), $e^{-\left(\frac{t}{\theta}\right)^{\beta}}$ is small for large values of $t$ and $\lambda_{s}(t)$ approximates the failure rate function of a single Weibull component. The result is similar to that of the two CFR components where the system failure rate asymptotically approaches CFR of a single component. Intuitively, the longer the system operates, the more likely it is that one of the components will fail, thus reducing the system to a single component. Based on Equation (12), we can estimate the design life by 48 seconds for a desired $90 \%$ reliability in our test environment with two randomly walking subjects, which increased around $40 \%$ as compared to a single component.

Figure 7 illustrates the redundant system with video sequences. A subject tracked by camera 1 is circled in red and a subject tracked by camera 2 is circled in yellow in Figure 7. Figure 7(a) demonstrates a case where both subjects are always tracked by both cameras. Figure $7(\mathrm{~b})$ demonstrates a case where one of the two subjects is not tracked by camera 1 at $t_{0}+10$ and $t_{0}+39$, but it is still tracked by camera 2. Similarly, one of the two subjects is not tracked by camera 2 at $t_{0}+21$, but it is still tracked by camera 1 . This experiment demonstrates that the redundant system does not loose the track of subjects. As a result, this redundant system can accomplish higher reliability. The procedure of how to identify the same subjects in both cameras, namely consistent labeling, is beyond the scope of this paper. Calderara's method [3] is applied in our experiment.

\section{Comparison}

In order to demonstrate our proposed framework has comparable results of existing approach, Nawaz and Cavallaro's approach [8] is used. We have four different trials and two objects in the experiment. Trails 1 and 2 present a single 
Table 3. Mean $(\mu)$ and standard deviation $(\sigma)$ based on Nawaz and Cavallaro's approach for all the trials

\begin{tabular}{|c|c|c|c|}
\hline Trial & Target & Cui's approach $(\mu)$ & Cui's approach $(\sigma)$ \\
\hline \multirow[t]{2}{*}{1} & 01 & 0.811 & 0.071 \\
\hline & $\mathrm{O} 2$ & 0.799 & 0.003 \\
\hline \multirow[t]{2}{*}{2} & 01 & 0.346 & 0.091 \\
\hline & $\mathrm{O} 2$ & 0.451 & 0.112 \\
\hline \multirow[t]{2}{*}{3} & 01 & 0.832 & 0.101 \\
\hline & $\mathrm{O} 2$ & 0.811 & 0.178 \\
\hline \multirow[t]{2}{*}{4} & 01 & 0.411 & 0.123 \\
\hline & $\mathrm{O} 2$ & 0.245 & 0.012 \\
\hline
\end{tabular}

tracking system experiment. Trails 3 and 4 present a redundant tracking system experiment. In trail 2, the experiment is conducted less than 34 seconds, which is derived by Equation (10). In trail 1, the experiment is conducted more than 34 seconds. In trail 4, the experiment is conducted less than 48 seconds, which is derived by Equation (12). In trail 3, the experiment is conducted more than 48 seconds. Whenever the two subjects are not being tracked simultaneously by the system, we stop the experiment. This procedure is carried out repeatedly during the experiment. We repeat the experiment for 40 times in order to collect respective mean and standard deviation numbers. Table 3 illustrates the experimental results. In table 3, it shows a redundant tracking system is more reliable than a single tracking system. In a single tracking system, the system is more reliable when it is operated less than 34 seconds. In a redundant tracking system, the system is more reliable when it is operated less than 48 seconds. In both cases, it shows comparable results as our proposed approach. Nevertheless, Nawaz and Cavallaro's approach is unable to give the failure time, which is important for the aspect of system design. Additionally, Nawaz and Cavallaro's approach does not provide a quantitative alternative to improve the overall system reliability, and a unified and statistical index to evaluate the performance of automated video surveillance systems.

\section{Discussions}

In essence, we need to determine if we should implement redundancy to improve the overall system reliability or improve the reliability of inherent component design. In our case, the inherent component design indicates the tracking algorithm. Thus, we need to know what the maximal reliability is that a system with identically redundant components can provide. The objective is to find the number of redundant components, $n$, so that

$$
\begin{aligned}
& \max \left\{1-(1-R(t))^{n}\right\}, \\
& \text { subject to } n \times c \leq B,
\end{aligned}
$$

where $c$ is the cost of the component. $B$ is the budget available for additional effort to improve the reliability of the system. Based on Equation (15), we should take the redundancy as the alternative to improve the overall system reliability if the maximal reliability that a system with identically redundant components can provide is bigger than the improved component design itself at a given budget $B$. Vice versa, we should take the improvement of inherent component design as the alternative to improve the overall system reliability.

Moreover, the benefit of our proposed model is to provide a unified and statistical index to evaluate the performance of automated video surveillance systems. However, since the cause of failure in an automated video surveillance system is not limited to the case of two randomly walking subjects, two open topics in this area are: (1) multiple criteria can be added to test the failure of the system (drastic changes of illumination, changing distance between objects, the effect of $\mathrm{CPU}$ load). Accordingly, multiÂ $\infty$ dimensional probability density estimation (eq. multidimensional kernel density estimation) can be used to estimate the reliability model of the system. (2) The lack of completely available failure data and indeterminate nature of future events lead to the uncertainty problem. Thus, uncertainty analysis (eq. the combination of Maximum-Entropy Principle $[8,12]$ and Bayesian approach) of the derived reliability model is necessary, which can help to understand the applicability of the reliability model. 


\section{Conclusion}

Most existing performance evaluation methods appear to be deterministic methods where a robustness factor such as two or three-times the expected number of subjects or the strength of illumination would be considered in the design. This often results in overdesign or underdesign. In this paper, we proposed a framework to analyze the failure process and determine the time to failure in automated video surveillance systems. With the help of the reliability model, we are able to introduce a unified and statistical index to evaluate the performance of automated video surveillance systems. Based on our proposed framework, the uncertainty problem of a failure process caused by the system complexity, imprecise measurements of the relevant physical constants and variables, and the indeterminate nature of future events can be addressed accordingly. In addition, the proposed framework can provide a guideline of how to improve the reliability of an automated video surveillance system.

\section{References}

[1] Bashir F., Porikli F., Performance evaluation of object detection and tracking systems, In: Proceedings of the 9th IEEE International Workshop on Visual Surveillance and Performance Evaluation of Tracking and Surveillance (VS-PETS 06), New York, NY, USA, 2006

[2] Collins R.T., Lipton A.J., Kanade, T., Introduction to the special section on video surveillance, IEEE T. Pattern Anal., 22(8), 745-746, 2000

[3] Calderara S., Prati A., Vezzani R., Cucchiara R., Consistent labeling for multi-camera object tracking, In: The 13th International Conference on Image Analysis and Processing, Roli, F., Vitulano, S. (Eds.), Springer, LNCS 3617, 1206-1214, 2005

[4] Chau D.P., Bremond F., Thonnat M. Online evaluation of tracking algorithm performance, In: The Int. Conf. on Imaging for Crime Detection and Prevention, 1-6, 2009

[5] Cui Y., Samarasekera S., Huang Q., Greiffenhagen M., Indoor monitoring via the collaboration between a peripheral sensor and a foveal sensor, In: IEEE Workshop on Visual Surveillance, 2-9, 1998

[6] Doermann D. Mihalcik D., Tools and techniques for video performance evaluation, In: The 15th Int. Conf. on Pattern Recognition, vol. 4, 167-170, 2000

[7] Dodson B. Nolan D. Reliability engineering handbook, CRC Press, 1999

[8] Dai Y.-S., Xie M., Log Q., Ng S.-H., Uncertainty analysis in software reliability modeling by Bayesian approach with maximum-entropy principle, IEEE T. Software Eng., 33(11), 781-795, 2007

[9] Ebeling C.E., An introduction to reliability and maintainability engineering, McGraw-Hill, 1997

[10] Erdem C., Tekalp A., Sankur B., Metrics for performance evaluation of video object segmentation and tracking without ground-truth, IEEE Image Proc., 2, 69-72, 2001

[11] Jaynes C., Webb S., Steele R. M., Xiong Q., An open development environment for evaluation of video surveillance systems, In: The 3rd Int. Workshop on Performance Evaluation of Tracking and Surveillance, 2002

[12] Kapur J., Maximum-entropy models in science and engineering, John Wiley \& Sons, 1989

[13] Kasturi R., Goldgof D., Soundararajan P., Manohar V., Garofolo J., Bowers R., Boonstra M., Korzhova V., Zhang J., Framework for performance evaluation of face, text, and vehicle detection and tracking in video: data, metrics, and protocol, IEEE T. Pattern Anal., 31(2), 319-336, 2009

[14] Lazarevic-McManus N., Renno J., Jones G. A., Performance evaluation in visual surveillance using the F-measure, In: The 4th ACM Int. Workshop on Video Surveillance and Sensor-Networks, 45-52, 2006

[15] List, T., Bins, J., Vazquez, J., Fisher, R.B., Performance evaluating the evaluator, In: Proc. 2nd Joint IEEE Int. Workshop on Visual Surveillance and Performance Evaluation of Tracking and Surveillance, (VS-PETS), Beijing, 129-136, 2005

[16] Lei R., Xu L.-Q., Real-time outdoor video surveillance with robust foreground extraction and object tracking via multi-state transition management, Pattern Recogn. Lett., 27(15), 1816-1825, 2006

[17] Nawaz T., Cavallaro, A., PFT: A protocol for evaluating video trackers, IEEE Image Proc. 2011

[18] Schlögl T., Beleznai C., Winter M., Bischof H., Performance evaluation metrics for motion detection and tracking, In: The 17th Int. Conf. on Pattern Recognition, 4, 519-522, 2004 
Camera 1
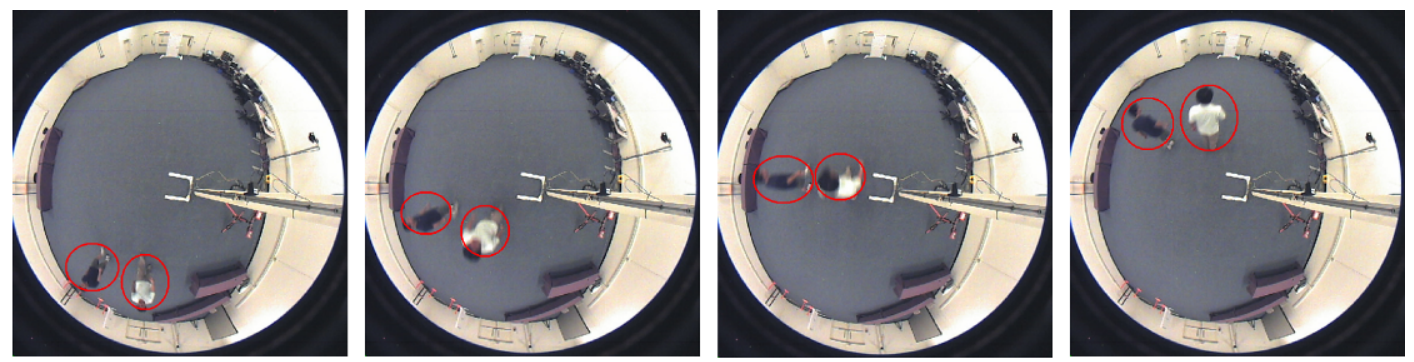

Camera 2

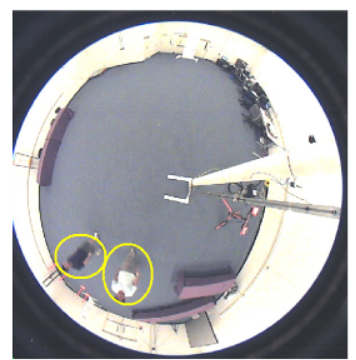

$t=t_{\theta}$ (seconds)

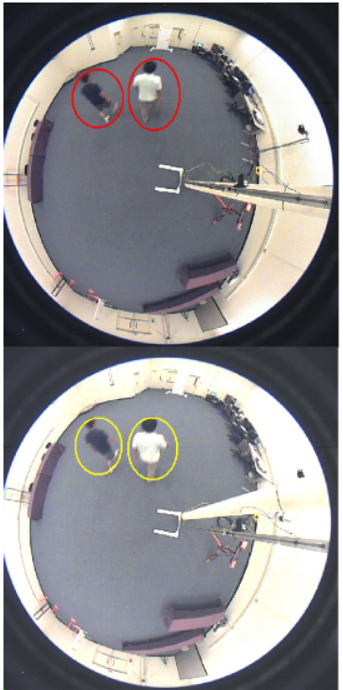

$t=t_{\theta}($ seconds $)$

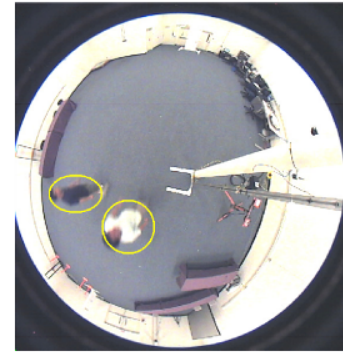

$t=t_{0}+14$ (seconds)

(a)

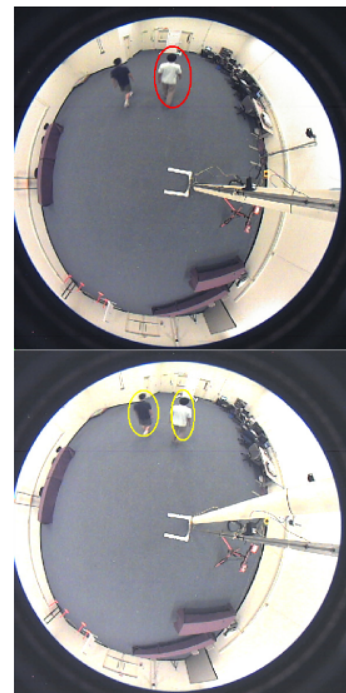

$t=t_{0}+10$ (seconds)

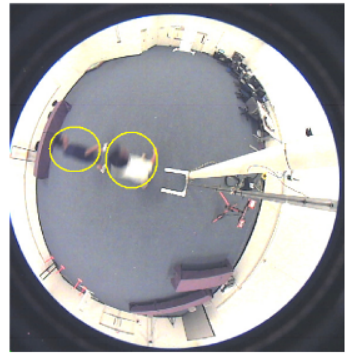

$t=t_{0}+26$ (seconds)

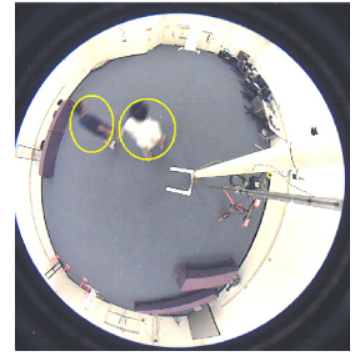

$t=t_{0}+45$ (seconds)
Camera 1

Camera 2

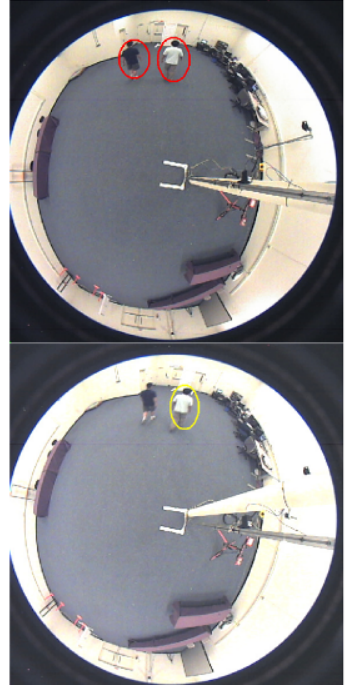

$t=t_{\theta}+21$ (seconds)

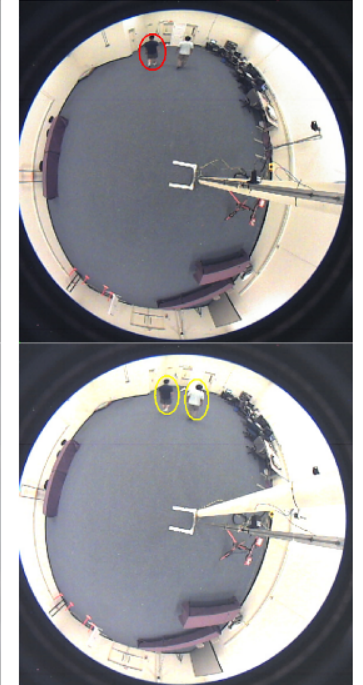

$t=t_{0}+39$ (seconds)

(b)

Figure 7. Illustration of video sequences in the redundant system. (a) two objects are completely tracked by both cameras. (b) at some time frames, one of those two objects is not tracked by either one of those two cameras, but it is still tracked by the other cameras. Thus, the redundant system does not lose the track of objects.

[19] Pan P., Porikli F., Schonfeld D., A new method for tracking performance evaluation based on a reflective model and perturbation analysis, IEEE ICASSP, 3529-3532, 2009

[20] Perera A. G. A., Hooqs A., Srnivas C., Brooksby G., Wensheng H., Evaluation of algorithms for tracking multiple objects in video, In: The 35th IEEE Applied Imagery and pattern Recognition Workshop, 35-35, 2006

[21] Popoola J., Amer A., Performance evaluation for tracking algorithms using object labels, Int. Conf. Acoust. Spee., 733-736, 2008

[22] Wackerly D.D., Mendenhall III W., Scheaffer R.L., Mathematical statistics with applications, 2nd edition, Duxbury 
Press, 2002

[23] Yilmaz A., Javed O., Shah M., Object tracking: a survey, ACM Comput. Surv. 38(4), 13, 2006

[24] Zhang M., Chen K., Guo Y.Y., Online parameter based Kalman filter precision evaluation method for video tracking, In: IEEE Int. Conference on Multimedia Technology, 598-601, 2011 